\title{
Myalgia, Obtundity and Fever in a Patient with a Prosthesis
}

\author{
$\underline{\text { Valeria Di Stefano }}{ }^{1}$, Margherita Migone De Amicis ${ }^{2}$, Cecilia Bonino $^{3}$, Natalia Scaramellini ${ }^{1}$, \\ Yvan Torrente $^{4}$, Stefania Piconi ${ }^{5}$, Francesca Minonzio ${ }^{2}$ \\ ${ }^{1}$ Department of Internal Medicine, Fondazione IRCCS Ca' Granda, Ospedale Maggiore Policlinico and Department of Clinical Sciences and Community \\ Health, University of Milan, Milan, Italy \\ ${ }^{2}$ Department of Internal Medicine, Fondazione IRCCS Ca' Granda, Ospedale Maggiore Policlinico, Milan, Italy \\ ${ }^{3}$ Department of Emergency Medicine, Fondazione IRCCS Ca' Granda, Ospedale Maggiore Policlinico, University of Milan, Milan, Italy \\ ${ }^{4}$ Stem Cell Laboratory, Department of Pathophysiology and Transplantation, University of Milan, Unit of Neurology, Fondazione IRCCS Ca' Granda \\ Ospedale Maggiore Policlinico, Centro Dino Ferrari, Milan, Italy \\ ${ }^{5}$ First Division of Infectious Diseases Unit, University of Milan, Ospedale L. Sacco, Milan, Italy
}

\section{Doi: 10.12890/2019_001021 - European Journal of Case Reports in Internal Medicine - ( ) EFIM 2019}

Received: $16 / 12 / 2018$

Accepted: $24 / 12 / 2018$

Published: 08/02/2019

How to cite this article: Di Stefano V, Migone De Amicis M, Bonino C, Scaramellini N, Torrente Y, Piconi S, Minonzio F. Myalgia, obtundity and fever in a patient with a prosthesis. EJCRIM 2019;6: doi:10.12890/2019_001021.

Conflicts of Interests: The Authors declare that there are no competing interests.

This article is licensed under a Commons Attribution Non-Commercial 4.0 License

\section{ABSTRACT}

Objective: We describe a rare case of group G streptococcus (GGS) sepsis complicated by bacterial toxin myopathy.

Case: A 65-year-old man, with a history of infection of his shoulder prosthesis, presented with multiorgan failure and notable myalgia likely caused by toxins. The patient was treated successfully with antibiotics and prosthesis removal.

Conclusion: This case suggests infection by GGS should be considered in a patient presenting with myalgia associated with sepsis.

\section{LEARNING POINTS}

- Infection by GGS should be considered in a patient presenting with myalgia associated with sepsis.

- The differential diagnosis in this case included a neurological condition (meningitis or atypical Guillain-Barré syndrome) and sepsis with myopathy induced by bacterial toxins.

- Group G streptococcus (GGS) infection in a prosthetic shoulder was successfully treated with antibiotics and prosthesis removal.

\section{KEYWORDS}

Group G streptococcus, streptococcal toxic shock syndrome, myopathy, myalgia, myositis

\section{CASE DESCRIPTION}

We present the case of a 65-years old patient hospitalized in February 2018 for fever, alteration of consciousness and arthromyalgia. He had a history of right shoulder replacement after traumatic fracture in 2011 and a diagnosis of persistent prosthesis infection and dislocation since 2015. Prosthesis replacement had been recommended by orthopaedics but had not been carried out.

In 2018, because of high persistent fever and diffuse pain, the patient was admitted to the Emergency Room and then to our Internal Medicine Unit. For some days before hospitalization, he had been taking non-steroidal anti-inflammatory drugs for lumbar pain without improvement.

On physical examination, the patient was obtunded, although responsive to verbal stimuli, and inclined to sleep. He presented normal vital parameters except for tachycardia and fever (BP 120/70 mmHg, HR 120/min, RR 18/min, O² Sat 93\%, temperature $38^{\circ} \mathrm{C}$ ). He complained of diffuse pain, mostly in the limbs, exacerbated by minimal movement and allodynia. Skin inspection demonstrated migratory skin erythema 
associated with generalized oedema and inflammatory signs on the right shoulder (calor, rubor, tumor, dolor e functio laesa). Neurological examination showed altered mental status and rigor nucalis. The chest and abdominal examination was normal.

Laboratory tests showed a raised inflammatory index (C-reactive protein $37 \mathrm{mg} / \mathrm{dl}$, procalcitonin $13 \mathrm{ng} / \mathrm{ml}$, erythrocyte sedimentation rate $108 \mathrm{~mm}$ ), creatine phosphokinase elevation (1,828 U/I) and multiorgan failure with renal, liver and lung dysfunction requiring oxygen therapy (serum creatinine $1.55 \mathrm{mg} / \mathrm{dl}$, urea $141 \mathrm{mg} / \mathrm{dl}$, aspartate transaminase $115 \mathrm{U} / \mathrm{l}$, serum cholinesterase 2,935 U/I, international normalized ratio 1.43 , serum albumin $2.5 \mathrm{~g} / \mathrm{dl})$. Blood gas analysis revealed an increased lactate level ( $3 \mathrm{mmol} / \mathrm{l})$.

A right shoulder x-ray confirmed prosthesis dislocation (Fig. 1).

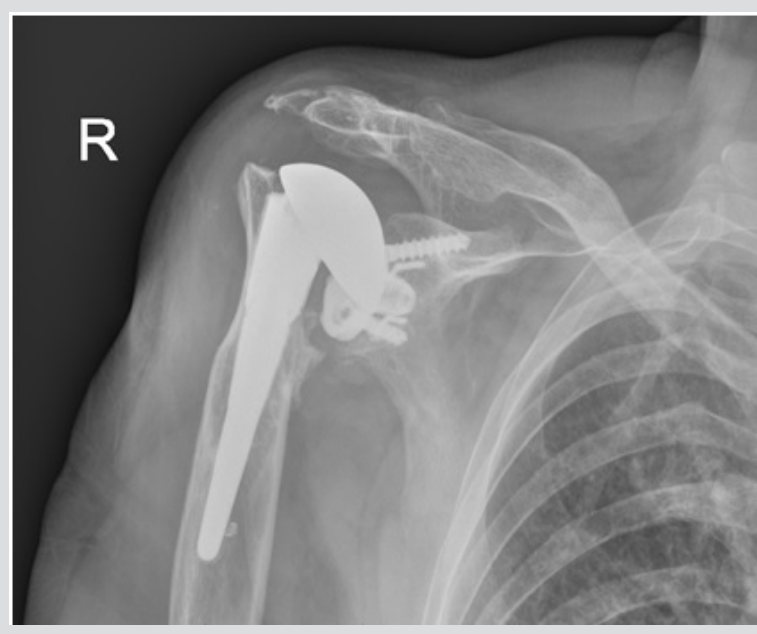

Figure 1. Right shoulder prosthesis dislocation

This presentation supported a neurological or a septic cause. Indeed, altered mental status, rigor nucalis and fever can suggest meningitis. Atypical Guillain-Barré syndrome, in which motor deficit is associated with pain in all four limbs, should also be considered. In contrast to typical Guillain-Barré syndrome, the motor deficit is not ascending and there are no deep tendon reflex abnormalities, dysautonomia, or respiratory or cranial nerve involvement. The presentation also indicated sepsis with multiorgan failure associated with myopathy.

In light of the history of right shoulder infection, arthrocentesis of the shoulder and blood cultures were first performed. Simultaneously we started fluid resuscitation and administered wide spectrum empiric antibiotics (ceftriaxone and vancomycin). As we were not able to perform lumbar puncture because of technical difficulties, we adjusted the ceftriaxone dose to cover for meningitis.

The results of blood and synovial fluid cultures showed infection with group $\mathrm{G} \beta$-haemolytic streptococcus. This finding led us to suspect myalgia as a result of damage by bacterial toxins. Therefore, vancomycin was replaced with clindamycin and immunoglobulin infusion was started.

A PET/CT scan and echocardiography excluded other sources of infection (Fig. 2).

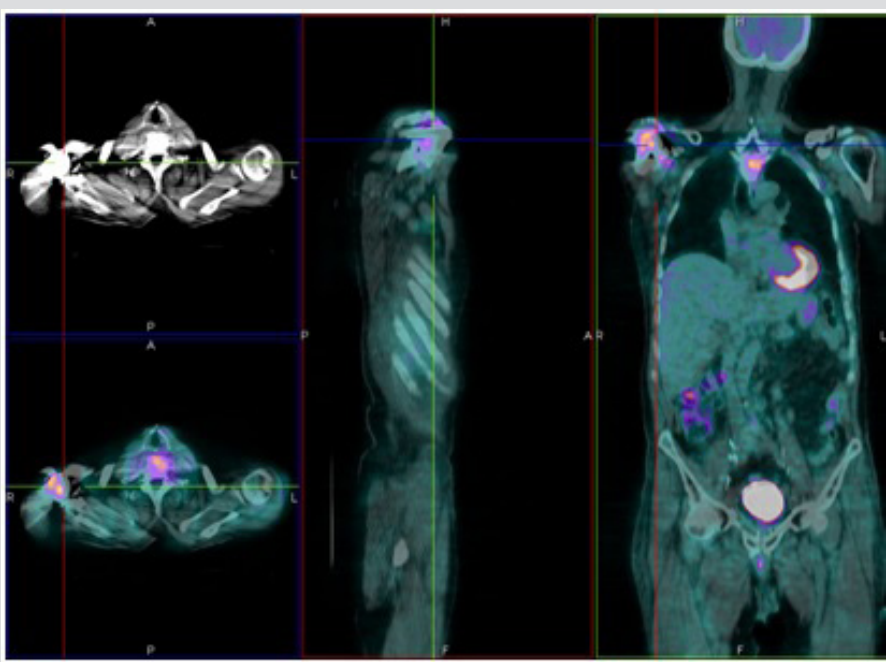

Figure 2. PET/CT scan showing a significant increase in glucose metabolism in right scapular-humeral periprosthetic bone, in contiguous soft tissues and in the right deltoid muscle 
Our patient began to recover after a few days of treatment, showing pain reduction and improvement in cognitive status. Electromyography (EMG) and electroneurography (ENG) showed moderate muscle pain in all four limbs, compatible with the diagnosis of bacterial toxininduced myopathy. The patient was scheduled for prosthesis removal after his clinical condition had stabilized.

\section{DISCUSSION}

Toxic syndrome due to group G streptococci (GGS) is rare and few cases have been described in the literature ${ }^{[1,2]}$. Diagnosis is based on clinical evidence, the inflammatory index and culture results.

GGS react with Lancefield group G typing serum and, similar to the well-known Streptococcus pyogenes (group A streptococcus, GAS), form large colonies on sheep blood agar, typically surrounded by a zone of $\beta$-haemolysis. GGS and GAS also have comparable virulence, sharing, for example, similar pyrogenic exotoxins implicated in streptococcal toxic shock ${ }^{[3]}$.

Streptococcal myositis is an unusual but particularly fulminant infection attributed to GAS; because GGS shares many similarities with GAS, it is may also be the aetiological agent in myositis ${ }^{[2]}$.

Exotoxins released by GGS act as superantigens activating the immune system by bypassing the usual antigen-mediated immune response sequence, resulting in the release of large quantities of inflammatory cytokines capable of mediating shock and tissue injury ${ }^{[1,4]}$. Moreover, exotoxins induce human mononuclear cells to synthetize TNF- $\alpha$, which could be the mediator of fever, shock and tissue injury ${ }^{[4]}$.

GGS, part of the normal commensal flora of the human upper airway, has been implicated in a wide range of human infections including septic arthritis. Consequently, the presence of a prosthetic joint is a predisposing factor for this condition. Non-steroid anti-inflammatory drugs, used also by our patient, are thought to predispose to severe forms of streptococcal infection by enhancing cytokine production induced by the exotoxins ${ }^{[1,4]}$.

Our patient did not have streptococcal toxic shock, as demonstrated by his stable blood pressure which remained within the normal range. However, signs of soft tissue infection (swelling and erythema) were present. When such manifestations occur in GAS toxic-shock syndrome, a high percentage of cases (70\%) progress to myositis ${ }^{[4]}$. Nevertheless, data on GGS infection are lacking.

This case is of interest because it suggests the differential diagnosis of meningitis, Guillain-Barré syndrome or bacterial toxin myopathy. As previously mentioned, we decided not to perform a lumbar puncture because the patient was compromised and in such a septic condition, the risks of procedure-induced infection are high. Moreover, our patient was already receiving proper antibiotic coverage.

We decided to perform EMG/ENG, which confirmed the hypothesis of peripheral muscular involvement. This, together with the improvement in the septic condition, despite the persistence of rigor nucalis and myalgias, retrospectively excluded the diagnosis of meningitis. Additionally, the EMG/ENG excluded atypical Guillain-Barré syndrome because no signs of active denervation were found.

Finally, in light of the septic shock with cutaneous erythema, generalized oedema, myalgia and leg and arm hyposthenia, we considered streptococcus toxin damage might be the cause of this clinical presentation. EMG/ENG confirmed this hypothesis, showing muscle disorders compatible with the diagnosis of polymyositis.

Following final diagnosis, the pharmacological approach was based mainly on antibiotic treatment: we used two drugs with a synergic effect: a $\beta$-lactam (ceftriaxone) which inhibits bacterial wall synthesis, and clindamycin which suppress bacterial toxin synthesis and penicillinbinding protein synthesis ${ }^{[4]}$.

Although successful treatment of GGS that included immunoglobulin therapy has been reported in the literature ${ }^{[3]}$, immunoglobulin infusion is still controversia[ ${ }^{[1]}$. A clear mechanism of action has not been shown and many hypotheses are described in the literature: streptococcal toxin neutralization, B lymphocyte proliferation inhibition, and TNF- $\alpha$ and IL- 6 inhibition.

In this case we chose to administer immunoglobulin because of the severe clinical condition of the patient.

\section{REFERENCES}

1. Barnham MRD, Weightman NC, Anderson AW, Tanna A. Streptoccocal toxic shock syndrome: a description of 14 cases from North Yorkshire, UK. Clin Microbiol Infect 2002;8:174-181.

2. Wagner JG, Schlievert PM, Assimacopoulos AP, Stoehr JA, Carson PJ, Komadina K. Acute group G streptococcal myositis associated with streptococcal toxic shock syndrome: case report and review. Clin Infect Dis 1996;23:1159-1161.

3. Baxter M, Morgan M. Streptococcal toxic shock syndrome caused by group G streptococcus, United Kingdom. Emerg Infect Dis 2017;23:127-129.

4. Stevens DL. Streptococcal toxic-shock syndrome: spectrum of disease, pathogenesis, and new concepts in treatment. Emerg Infect Dis 1995;1:69-78. 